\title{
Modelling and Performance Impact of High Power/High Energy Battery Hybrids on Fixed-Wing eVTOL UAV
}

\author{
Philipp Stahl ${ }^{1}$, Christian Roessler ${ }^{2}$, Mirko Hornung ${ }^{3}$ \\ Technical University of Munich, Garching, 85748, Germany
}

\begin{abstract}
Fixed-wing eVTOL UAV are unmanned aerial vehicles with electric powertrains capable of both hover and fast wing-borne forward flight. The high power consumption of the electric powertrains during vertical flight is in conflict with the usage of high energy density batteries. This study suggests dual battery architectures assembled from both high energy and high power battery types and with different complexity levels to overcome this problem. A modelling approach on the fidelity level of conceptual aircraft is presented. In an exemplary study with different aircraft types, the battery architectures' impact on aircraft performance is examined. With a single battery architecture as baseline, the results show improvements in endurance of 10 to $20 \%$ as a retrofit solution with current battery technology.
\end{abstract}

\section{Introduction}

Fixed-wing VTOL aircraft operate in a powered lift 'hover' mode for take-off and landing and in a wing-borne forward flight 'cruise' mode. This provides the ability of take-off and landing in confined environments and, at the same time, fast and efficient cruise flight. Applications are urban air mobility in the manned sector and various forms of surveillance or cargo transport in the unmanned sector, on which this study focuses. The use of electric powertrains simplifies the implementation of distributed propulsion, reduces powertrain complexity and maintenance effort and supports low noise signatures. The usage of a battery as power supply is confronted with both challenges and chances compared to pure VTOL or CTOL vehicles.

Fig. 1 shows the inherent trade in battery design between energy density and power density, together with typical power requirements of the flight phases 'cruise', 'cruise climb', 'hover' and 'hover climb'. The achievable energy density, and with that endurance and range, for a VTOL vehicle is significantly reduced of what is useable for a CTOL aircraft as there is no 'hover' or 'hover cruise' requirement. For fixed-wing VTOL vehicles, the time share of VTOL phases is less than $10 \%$ of overall mission time. Hence, only a small portion of the energy must be provided at the highest discharge rates. This opens up the opportunity to compose the battery system of multiple batteries that are tailored to the individual power and energy requirements. The goal is to maximize the overall useable energy density of the battery system.

The presented research examines different kinds of battery architectures. Besides aspects regarding safety, operational constraints and practical implementation, mainly flight performance is evaluated. Therefore the battery architectures' modelling as part of a fixed-wing VTOL UAV conceptual design tool is addressed.

\footnotetext{
${ }^{1}$ Research Scientist, Institute of Aircraft Design, philipp.stahl@tum.de

${ }^{2}$ Supervisor, Institute of Aircraft Design, christian.roessler@tum.de

${ }^{3}$ Professor, Institute of Aircraft Design, mirko.hornung@tum.de
} 


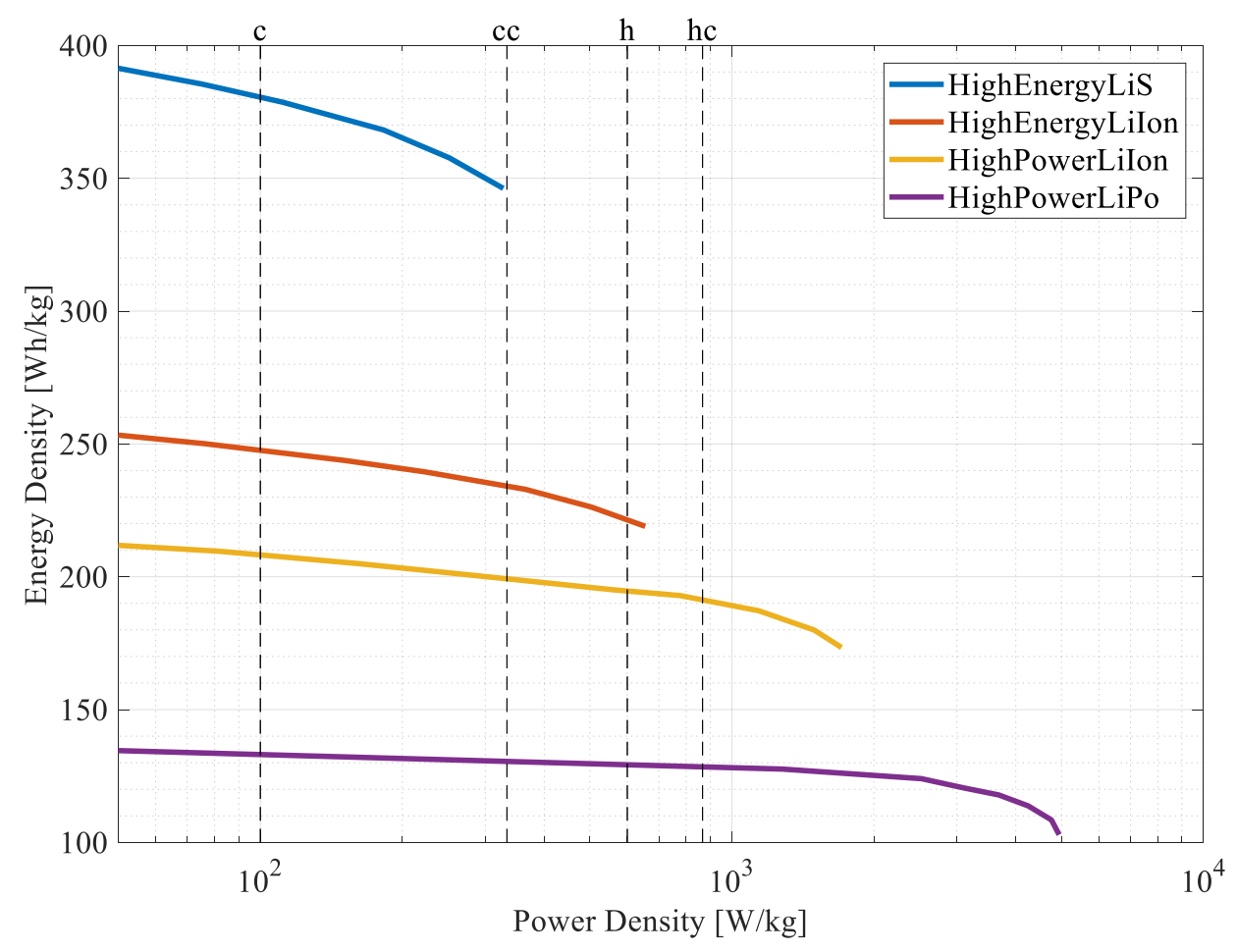

Fig. 1 Energy density vs. power density for different battery types (on cell level).

\section{Battery Architectures}

Fig. 2 illustrates the examined battery architectures. The considered battery architectures are integrating a maximum of two batteries with two powertrain groups. The batteries are characterized by their energy and power density. BAT 1 owns the higher energy density while BAT 2 provides the higher power density. The powertrain group CPT/DMPT (cruise powertrain/dual-mode powertrain) contains powertrains either exclusively used in the cruise phases, or used in both hover and cruise phases. The HPT (hover powertrain) group is only active in hover phases.

‘SGL BAT'

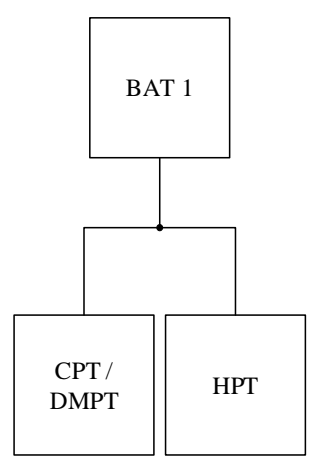

'SPRTD BAT'

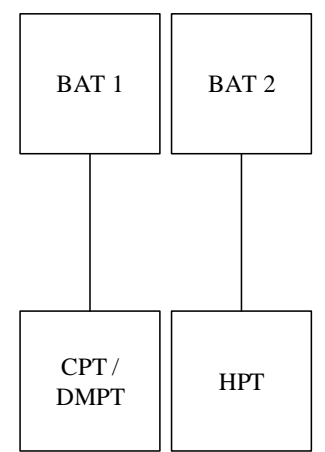

'BAT MUX'

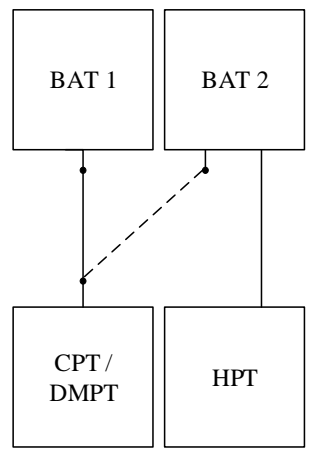

'BPMS BAT 1'

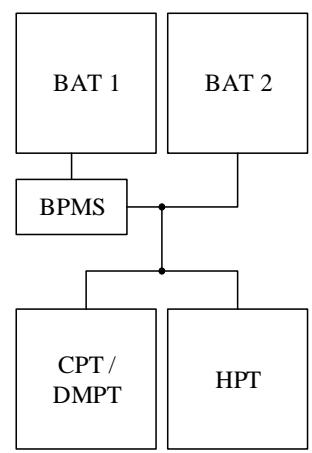

'BPMS BAT 2'

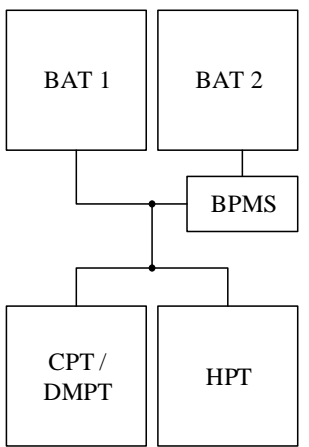

Fig. 2 Investigated battery architectures.

SGL BAT uses a single battery of one battery type that supplies both hover and cruise power requirements of all powertrains. This is the simplest implementation of a battery power supply. Other than with all other architectures, all energy can be spent on hover phases. Hence, it provides the highest flexibility for applications with extended hover times.

SPRTD BAT uses separated batteries for the cruise and hover powertrain. Due to its lower power drain, the cruise powertrain is connected to the high energy battery. It represents the simplest two-battery architecture. Benefits regarding the probability of a complete power loss emerge. 
BAT MUX extends the latter architecture by a multiplexer switch to enable energy crossfeed. According to the power capability, a crossfeed is only possible from the high power battery to the cruise powertrain. Redundancy against the failure of the high energy battery is introduced. An additional switch must be considered in the cost, mass and failure probability breakdown.

BPMS BAT 1 uses a battery power management system that is connected to the high energy battery (analog to [1]). It limits the power drained from the high energy battery to the allowable rate. The rest of the required power is provided by the high power battery. With that, a maximum in energy for all powertrains and flight phases can be stored weightefficiently in the high energy density battery. The battery power management system can further be used to recharge/keep charged the high power battery during low power flight phases. Again additional cost, mass and failure modes have to be considered. Losses in the battery power management system reduce the energy effectively useable from the high energy battery. Off-the-shelf devices used for battery charging can be adopted to serve as the battery power management system.

BPMS BAT 2 moves the battery power management system to the high power battery. It again limits the maximum power drain of the high energy battery to the allowed maximum. Compared to BPMS BAT 1, less energy passes through the battery power management system. Associated losses are reduced. The feasibility of this type of battery power management system still has to be proven.

The share of power between the batteries is independent of the powertrain architecture for both architectures with power management systems and the single battery architecture.

In principle, all dual battery architectures allow recharging the high power battery from the high energy battery using an onboard battery charger. This is however not further evaluated in this study. As dual battery architectures promote the usage of more energy dense battery types, the charge current rates are reduced. The increased charge times as well as the general handling of two instead of one battery may be detrimental to some applications.

\section{Battery System Modelling}

To evaluate the performance impact of the battery architectures within the complete aircraft context, the battery architectures are implemented as part of a fixed-wing VTOL conceptual aircraft design tool [2] [3]. Hence, the applied battery modelling techniques suit the fidelity level and computational speed requirements of conceptual design. Nevertheless, the battery is one of the most performance driving systems for electric, especially electric VTOL aircraft. The following models try to find a good balance of fidelity and effort for conceptual design.

\section{A. Energy vs. Power Trade}

The curves in Fig. 1 show the trade between energy density and power density on two levels.

1) By the choice of the battery cell type, energy storage capability is traded against power provision capability.

2) For each battery cell type, useable energy is diminished with increasing discharge rate.

With commercially available battery types, only discrete choices along the energy-power constraint are possible. Nominal energy density is calculated from discharge tests under little current. Maximum power density derives from the manufacturer's maximum discharge current rating and the corresponding voltage at minimum state of charge. This ensures that all aircraft performance requirements are met at minimum state of charge. A factor $\mathrm{f}_{\text {pack }}$ (typ. 110\%) is applied to the cell mass to account for battery pack assembly.

The dependency of useable energy and discharge rate is pronounced for high energy-density battery cells and can lead to energy losses up to $15 \%$. The losses are mainly caused and modelled by the internal resistance of the battery cell [4]. In this work however, this effect is covered to its complete extent by the battery efficiency $\eta_{\text {bat }}$.

$$
\eta_{\text {bat }}=\frac{E_{b a t}(P)}{E_{b a t}(P \rightarrow 0)}
$$

It relates the useable battery energy due to high power drain with the useable battery energy for the hypothetical 'zero power' drain case. In that way, each mission segment (which is assumed to have constant power drain) gets assigned its battery efficiency $\eta_{\text {bat }}$. Battery data containing the battery efficiencies are generated from discharge curves and stored as lookup tables. The applied model uses power drain instead of the usual current drain as the source of useable battery energy degradation. Dependent on the state of charge voltage, the same power can be provided at different currents and affect energy degradation differently. This inaccuracy is compensated by calculating a mean battery efficiency which represents the complete battery discharge cycle.

For battery life extension, the battery energy is not used to its full extent (typ. 80\%). 


\section{B. Discrete Cell Sizing}

The conceptual aircraft design tool takes the desired total aircraft mass as an input. After subtracting system, structural and payload mass items, the battery mass budget remains. This budget must be exploited to a best possible extent to maximize onboard energy. Due to the high capacity of best-performing battery cells in relation to the total battery pack capacity of small unmanned aircraft, a battery pack only consists of few parallel cell strings. Additionally a low cell voltage compared to the required powertrain voltage supports this effect. Consequently, the energy steps in which battery packs can be manufactured are significant. E.g. going from a $4 p$ to the next bigger $5 p$ cell configuration means $+25 \%$ in energy and mass. Therefore, batteries are discretely sized respecting possible cell interconnections. The popular 'rubber sizing' method for batteries can lead to significant mistakes. It disrespects to which extent the battery mass budget can be used by a feasible battery with discrete energy steps.

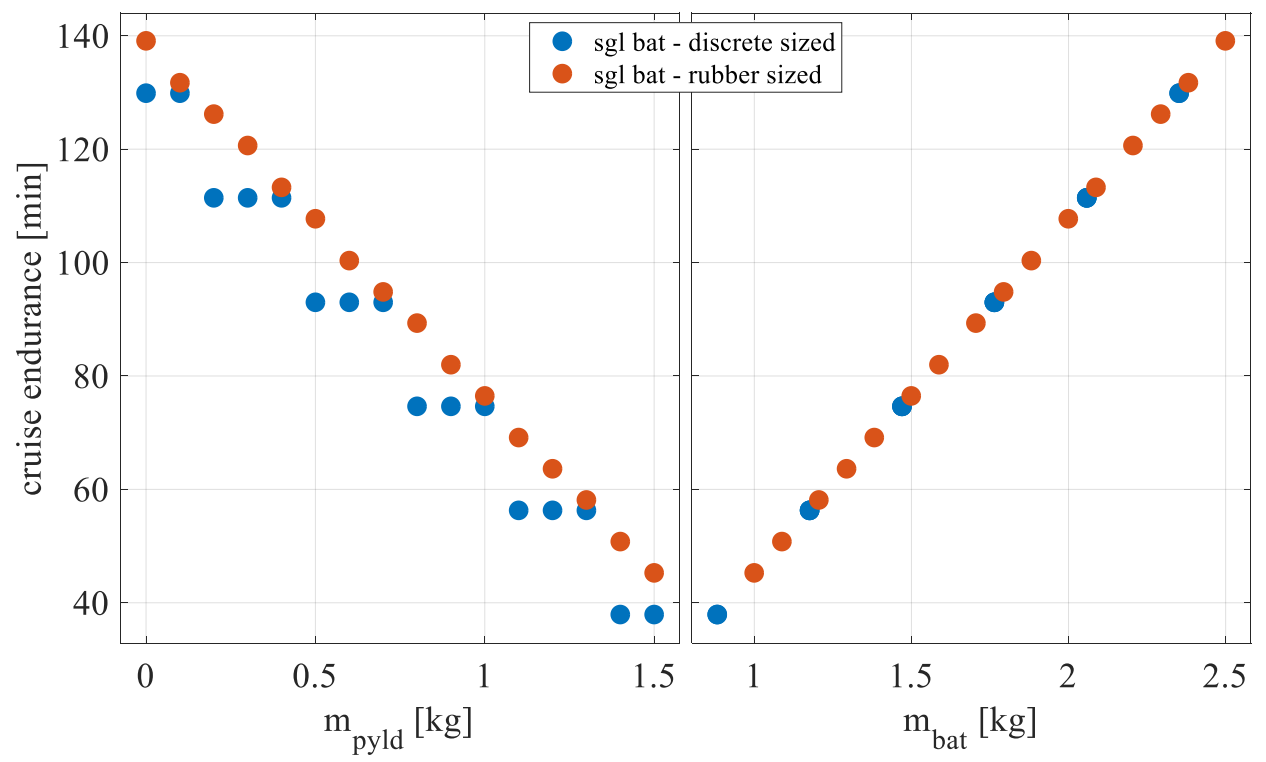

Fig. 3 Discrepancy between discrete and rubber battery sizing.

Fig. 3 depicts the mistakes made by battery rubber sizing in a payload-endurance diagram for a $5 \mathrm{~kg}$ fixed-wing VTOL UAV with a single battery architecture. The discrepancy in endurance reaches well beyond $33 \%$ for high payloads respectively small battery mass budgets.

\section{Battery Architecture Modelling}

All battery architectures are covered with the same set of equations. This leads to a framework that allows to

1) attach different power consumptions to each battery

2) share power consumptions among the batteries

3) enable and disable energy crossfeed

4) insert efficiencies of devices like switches or battery power managements systems

for each mission phase. This framework can even be adopted for fuel-electric hybrid power sources. Due to the shape of the problem, a 'try and error' approach is used. This means that the discrete combinations of the two batteries are calculated and checked later for their validity.

To reduce the number of battery combinations to be calculated, only parallel cell counts $n_{p, i}$ (with $i$ representing battery 1 and 2) that fit within the total battery mass budget and only reasonable combinations of cell types type $e_{i}$ are considered. For exmaple, battery type 1 should have a higher energy density than battery type 2 . Battery masses $\mathrm{m}_{\mathrm{i}}$, internal battery energy $E_{i}$ and maximum power capability $P_{i}$ calculate using

$$
\begin{gathered}
\mathrm{m}_{\mathrm{i}}=\mathrm{n}_{\mathrm{s}, \mathrm{i}} \mathrm{n}_{\mathrm{p}, \mathrm{i}} \mathrm{m}_{\text {cell,type }_{\mathrm{i}}} \mathrm{f}_{\text {pack }} \\
\mathrm{E}_{\mathrm{i}}=\left.\mathrm{m}_{\mathrm{i}} \frac{\mathrm{E}}{\mathrm{m}}\right|_{\text {pack,type }_{\mathrm{i}}}
\end{gathered}
$$




$$
P_{i}=\left.m_{i} \frac{P}{m}\right|_{\text {pack,type }_{i}}
$$

The serial cell count $\mathrm{n}_{\mathrm{s}, \mathrm{i}}$ derives from a predefined powertrain voltage. Power drains $\mathrm{P}_{\mathrm{i} \text {,phase }}$ from the batteries are calculated for each mission phase ('hover climb', 'cruise climb', 'cruise', 'cruise descent', 'hover descent'). An optional 'hover' phase can be included. If the battery architecture allows for a crossfeed, the energy of the 'hover' phase can be used for cruise as well. It increases the flexibility of a battery system to supply extended hover. The energy of the flight phase 'hover reserve' is only used in case of unexpected scenarios like landing site blockage. For UAV applications, a hover reserve is sufficient. Manned applications should include a cruise reserve as well.

$$
\begin{gathered}
\mathrm{P}_{1, \text { phase }}=\min \left(\mathrm{k}_{\text {share }, 1} \mathrm{P}_{1}, \frac{\mathrm{P}_{\text {load }, 1}}{\eta_{\mathrm{BPMS}, 1}}\right) \\
\mathrm{P}_{2, \text { phase }}=\frac{\max \left(0, \mathrm{P}_{\text {load }, 2}-\mathrm{k}_{\text {share }, 2} \mathrm{P}_{1} \eta_{\mathrm{BPMS}, 1}\right)}{\eta_{\mathrm{BPMS}, 2}}
\end{gathered}
$$

Table 1 Parameters to control the battery calculation.

\begin{tabular}{c|c|c|c|c|c|c|c|c|c} 
& \multicolumn{2}{|c|}{$\eta_{\text {BPMS }}$} & \multicolumn{2}{c|}{$\mathrm{k}_{\text {share }}$} & \multicolumn{2}{c|}{ Pload, } & \multicolumn{2}{|c|}{ Pload,2 } & $\mathrm{k}_{\text {xfeed }}$ \\
\cline { 2 - 10 } & 1 & 2 & 1 & 2 & h,hres,hchd & c,cc,cd & h,hres,hc,hd & c,cc,cd & \\
\hline SGL BAT & 1 & 1 & $\infty$ & 0 & all pwtr & all pwtr & 0 & 0 & 0 \\
\hline SPRTD BAT & 1 & 1 & $\infty$ & 0 & $\mathrm{dmpt}$ & $\mathrm{dmpt} / \mathrm{cpt}$ & $\mathrm{hpt}$ & 0 & 0 \\
\hline BAT MUX & 1 & 1 & $\infty$ & 0 & $\mathrm{dmpt}$ & $\mathrm{dmpt} / \mathrm{cpt}$ & $\mathrm{hpt}$ & $\begin{array}{c}\mathrm{c}: \mathrm{dmpt} / \mathrm{cpt} \\
\mathrm{cc}, \mathrm{cd}: 0\end{array}$ & 1 \\
\hline BPMS BAT 1 & $\eta_{\text {BPMS }}$ & 1 & 1 & 1 & all pwtr & all pwtr & all pwtr & all pwtr & 1 \\
\hline BPMS BAT 2 & 1 & $\eta_{\text {BPMS }}$ & 1 & 1 & all pwtr & all pwtr & all pwtr & all pwtr & 1
\end{tabular}

Table 1 controls the parameters dependent on the battery architecture. The battery power management system

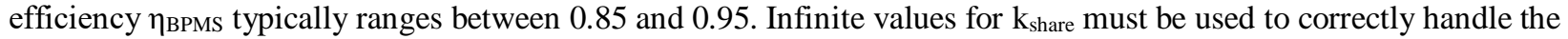
min statement in Eq. (5). Min and max statements are required for architectures with battery power management system. The variables $\mathrm{P}_{\text {load, } \mathrm{i}}$ are assigned with the power consumptions of the physically connected powertrains of the respective mission phase. For simplicity, only powertrain powers and no system or payload powers are listed. The present BAT MUX parameters assume aircraft configurations that use powertrains being exclusively active in hover (hpt) together with dual-mode powertrains (dmpt) or cruise-only powertrains (cpt) for the architecture. For aircraft configurations that only use dual-mode powertrains for hover the BAT MUX parameters have to be adapted. $\mathrm{k}_{\mathrm{xfeed}}$ is 1 if a power crossfeed from high power battery to high energy battery is possible. The required energies $\mathrm{E}_{\mathrm{i}, \mathrm{phase}}$ are calculated using the predefined times $t_{\text {phase }}$ for all phases except cruise.

$$
E_{i, \text { phase }}=\frac{P_{i, \text { phase }} t_{\text {phase }}}{\eta_{\text {bat,i,phase }}}
$$

The energies $\mathrm{E}_{\mathrm{i}, \mathrm{c}}$ can be spent on the 'cruise phase'. They and the associated cruise endurances $\mathrm{t}_{\mathrm{i}, \mathrm{c}}$ are calculated using the maximum allowed degree of discharge $\mathrm{DoD}_{\max }$ and the beforehand calculated energies $\mathrm{E}_{\mathrm{i}, \text { phase }}$ of time-fixed phases.

$$
\begin{gathered}
E_{i, c}=D_{\text {max }} E_{i}-\sum_{\substack{\text { phase }=\\
\text { h,hres(if no xfeed),hc,hd,cc,cd }}} E_{i, \text { phase }} \text { while } E_{2, c}=0 \text { for } k_{x f e e d}=0 \\
t_{i, c}=\frac{E_{i, c} \eta_{\text {bat,i,c }}}{P_{i, c}}
\end{gathered}
$$


The total cruise endurance results in

$$
t_{c}=\sum_{i=1,2} t_{i, c}
$$

To calculate the mass of the multiplexer or battery power management system, a respective mass-per-power factor together with the maximum conducted power is used. The following filters are applied to the battery combinations to guarantee validity.

$$
\begin{gathered}
\mathrm{P}_{\mathrm{i}}>\mathrm{P}_{\mathrm{i}, \text { phase }}>\sum_{\substack{\text { phase }=\\
\text { h,hres,hc,hd,cc,cd }}} \mathrm{E}_{\mathrm{i}, \text { phase }} \\
\mathrm{m}_{\text {bat,budget }}>\sum_{\mathrm{i}=1,2, \mathrm{MUX} / \mathrm{BPMS}} \mathrm{m}_{\mathrm{i}}
\end{gathered}
$$

For applicable architectures, the conditions in Eq. (11) and (12) need to be modified if it is desired to let battery 2 compensate a battery 1 failure. Among the valid battery combinations of each battery architecture, the one providing the highest cruise endurance $t_{c}$ is selected.

\section{Battery Architecture Performance Evaluation}

\section{A. Target and Boundary Conditions}

An exemplary study is performed to figure out the battery architectures' impact on mission performance. As target for the embedded optimizations and the measure for the mission performance, the endurance of the cruise phase is used.

Table 2 summarizes the key parameters that describe the aircraft and mission in the example study. Battery systems are designed to be capable of additional $60 \mathrm{~s}$ of hover. This capability is not used in the example mission. The related energy is spent in cruise endurance extension as far as the battery architecture allows for it. Battery 2 is not sized to compensate a failure of battery 1 with any of the battery architectures.

\section{Table 2 Parameters for the example study.}

\begin{tabular}{c|c} 
aircraft mass $[\mathrm{kg}]$ & 5 \\
\hline time for reserve hover [s] & 30 \\
\hline time for additional hover capability [s] & 60 \\
\hline time in 'hover climb'/'hover descent'/'cruise climb'/'cruise descent' [s] & $17 / 50 / 67 / 67$ \\
\hline BPMS efficiency [-] & 0.9 \\
\hline maximum degree of discharge [-] & 0.8 \\
\hline mass factor for battery pack assembly [-] & 1.1 \\
\hline mass-to-power factor for BPMS/MUX [10 $\left.{ }^{-4} \mathrm{~kg} / \mathrm{W}\right]$ & $1.7 / 0.85$
\end{tabular}

Fig. 4 shows the aircraft configurations used within the study. Both use dedicated powertrains exclusively in the hover phases. The QUAD uses a fifth powertrain exclusively for cruise propulsion. The rear powertrain of the TRI is a dual-mode powertrain which is active in both hover and cruise phases. Mainly due to the lower overall disk area of the hover involved powertrains, the TRI requires more power in hover. 

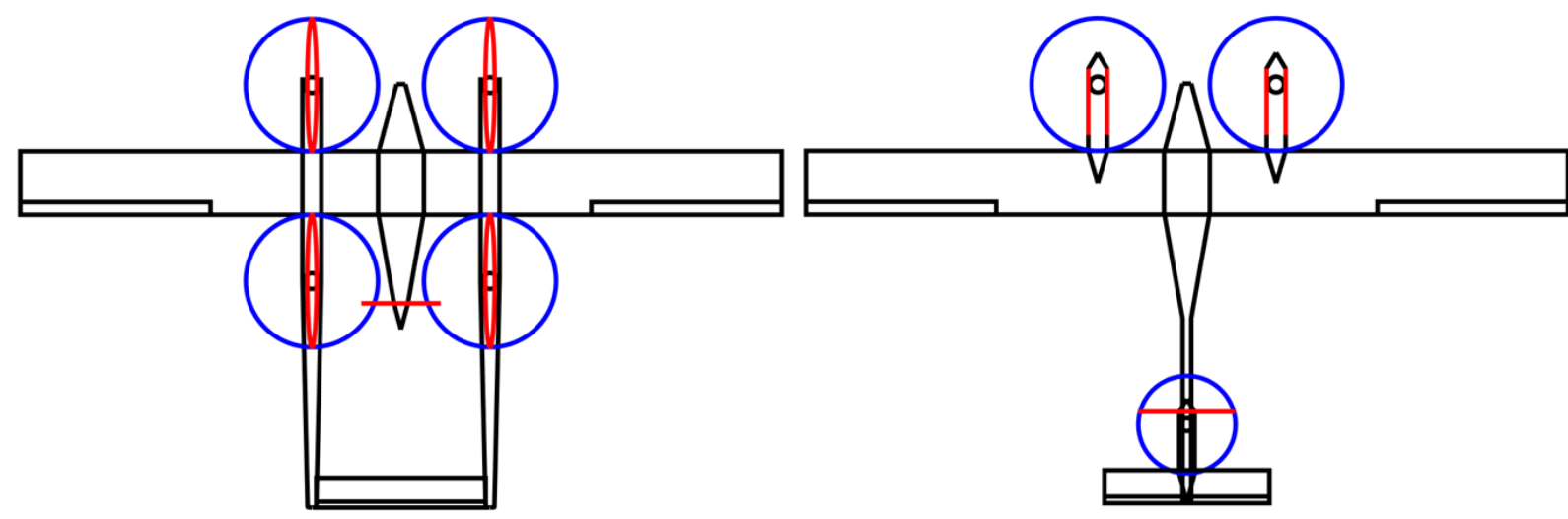

Fig. 4 Aircraft configurations used for the study: QUAD (left) and TRI (right).

Table 3 Considered battery cell types for the study.

\begin{tabular}{c||c|c|c|c} 
battery type number & 1 & 2 & 3 & 4 \\
\hline name & High Energy LiS & High Energy LiIon & High Power LiIon & High Power LiPo \\
\hline representative & $\begin{array}{c}\text { Oxis Energy } \\
\text { POA000412 }\end{array}$ & $\begin{array}{c}\text { Panasonic } \\
\text { NCR18650GA }\end{array}$ & $\begin{array}{c}\text { Sony } \\
\text { US18650VTC5 }\end{array}$ & $\begin{array}{c}\text { generic LiPo, 40C } \\
\text { discharge rating }\end{array}$ \\
\hline cell mass [g] & 85 & 47 & 44 & 10 (increment) \\
\hline capacity [Ah] & 14.7 & 3.5 & 2.6 & 0.35 (increment) \\
\hline nominal voltage [V] & 2.1 & 3.6 & 3.6 & 3.7 \\
\hline $\begin{array}{c}\text { nominal energy density of } \\
\text { cell [Wh/kg] }\end{array}$ & 400 & 260 & 214 & 135 \\
\hline $\begin{array}{c}\text { power density of cell at } \\
\text { end of discharge [W/kg] }\end{array}$ & 325 & 655 & 1700 & 4950
\end{tabular}

Table 3 and Fig. 1 contain the battery types among which the aircraft design tool can choose to build up the battery system. Panasonic's NCR18650GA [5] ranges among the most energy-dense lithium ion battery cells on the commercial market. Sony's NCR18650VTC5 [6] is a proven lithium ion battery cell in the high power segment. 'HighPowerLiPo' represents widely available lithium polymer battery cells as used in the remote control hobby sector (data from [7]). They are available in very small mass or capacity increments. 'HighEnergyLiS' represents a future battery generation promising significantly increased energy density. Data from Oxis Energy's POA000412 [8] prototype cells is used. Due to lack of data, the degradation of useable energy with increased power is adopted from the high energy lithium ion battery. The reduced voltage level and high cell mass must be noted.

\section{B. Results and Description}

In the following, the principle mechanisms acting during battery system selection are explained. Fig. 5 to Fig. 7 show the mass, power and energy breakdowns for the different battery architectures for the two aircraft configurations QUAD and TRI with a payload of $1 \mathrm{~kg}$. Except the battery system and the payload mass, no aircraft parameter were changed. The shown battery combinations yield the best cruise endurance for each battery architecture. The relevant characteristics of the aircraft configurations are the mass budget for the battery system, the power consumptions and the powertrain type. TRI's dual-mode powertrain takes power from the high energy battery 1 in hover while QUAD's hover powertrain only gets supplied by high power battery 2 (for architectures SPRTD BAT and BAT MUX). The battery mass budgets are almost identical for this payload mass (QUAD: $1.249 \mathrm{~kg}$, TRI: $1.253 \mathrm{~kg}$ ). QUAD's lower disk loading in hover yields less power consumption. In cruise, missing drag of inactive hover rotor blades make the TRI more efficient. 


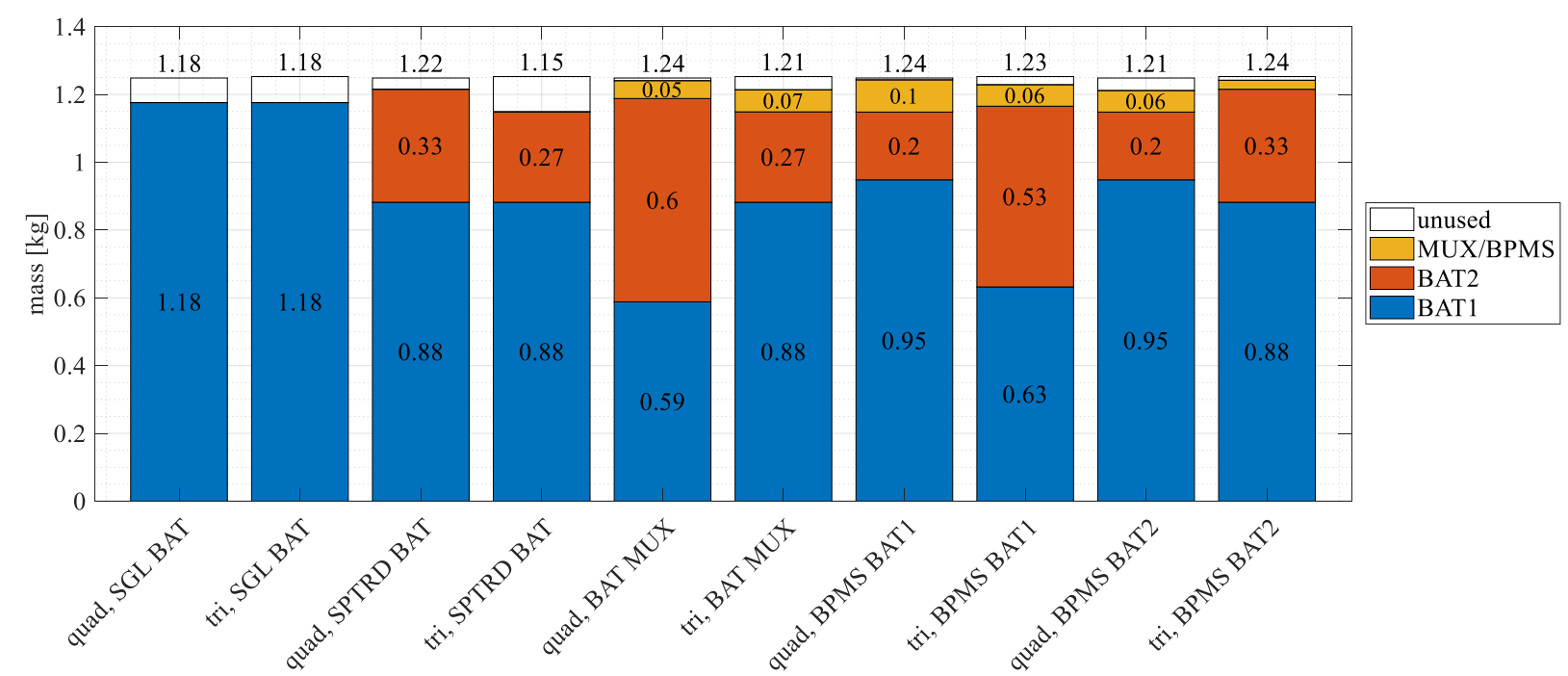

Fig. 5 Battery system mass breakdown.

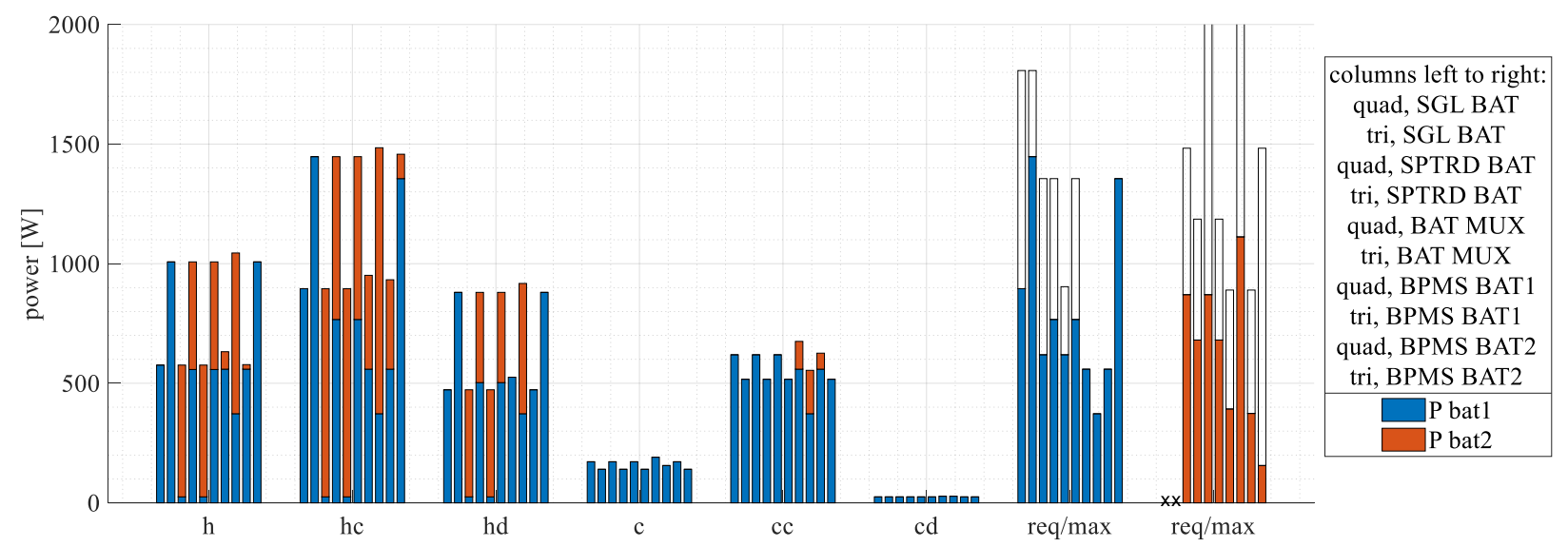

Fig. 6 Battery power drains for the flight phases $(h=$ hover, $h c=$ hover $\operatorname{climb}$, hd $=$ hover descent, $c=$ cruise, cc $=$ cruise climb, $c d=$ cruise descent $)$. White stack represents maximum power capability of battery.

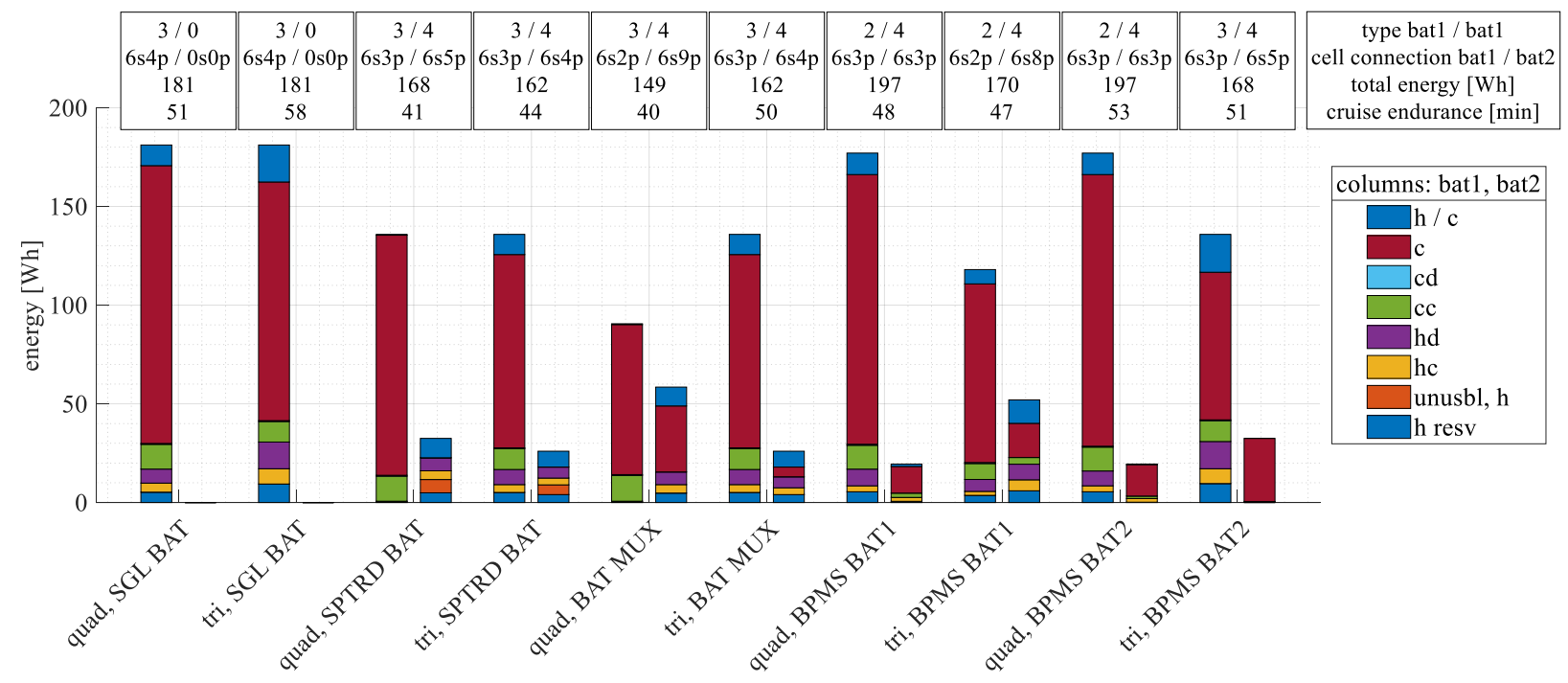

Fig. 7 Battery energy breakdowns by flight phases. 
Both aircraft can install the same amount and type of battery with SGL BAT. Due to the better cruise efficiency, the TRI can make up for the higher energy spent on the hover phases and achieve a longer cruise endurance. The ' $\mathrm{h} / \mathrm{c}$ ' energy which is planned for extended hover phases is useable for cruise with the SGL BAT architecture. For safety reasons, this is preferably done after the normal cruise energy is spent. Both aircraft achieve a good usage of the battery mass budget for this particular payload mass.

With SPRTD BAT, both aircraft use the same battery types for bat 1 and bat 2. TRI's dual-mode powertrain uses energy from bat 1 for hover which is stored with higher energy density than bat 2 . Despite some energy for systems and payload, QUAD takes all hover power and energy from bat 2. This increased energy need of the QUAD requires a bigger bat 2 than for the TRI. The general presence of a bat 2 forces bat 1 to shrink for both aircraft. The cruise endurance consequently is smaller than with SGL BAT. Increasing the number of parallel cells of bat 2 to fill the battery mass budget of the TRI is without benefit for the cruise endurance as no crossfeed to the cruise powertrain is possible. 'unusbl,h' energy, which either origins from discrete battery sizing or sizing for power, and ' $\mathrm{h} / \mathrm{c}$ ' energy remain unused unless an extended hover mission is flown.

BAT MUX forces to shrink bat 1 for the QUAD to fit the multiplexer switch mass within the budget. The cruise endurance however remains almost unchanged as crossfed bat 2 energy compensates. The battery budget is used almost to the full extent due to the small increment of battery type 4. For the TRI, the open mass budget of SPRTD BAT is enough to fit a multiplexer switch without reducing bat 1 . The now useable crossfed energy increases the cruise endurance by 6 minutes with regard to SPRTD BAT.

BPMS BAT 1 architecture allows to use the second most energy dense battery type for bat 1 . Per definition, the power capability of bat 1 is fully used. In hover climb, the leftover power for bat 2 is more than twice as high for the TRI as for the QUAD. The reasons are generally higher hover power consumption and the smaller bat 1 power capability. This forces TRI's bat 2 to be enlarged which requires bat 1 to shrink. Again, TRI's bat 2 is used to fill the battery budget best possible. Therefore, bat 2's power capability significantly exceeds its power requirement. Generally, the power drains for the flight phases are increased over the non-BPMS architectures. Given the same power arriving to the powertrains, the BPMS's efficiency increases the power drains from the batteries.

BPMS BAT 2 conducts less power through the BPMS. Its mass and power losses are reduced. For the QUAD this results in a 5-minute increase in cruise endurance using the same battery combination as in BPMS BAT 1. Also, the QUAD's endurance exceeds the SGL BAT benchmark endurance. For TRI, power from bat 2 only has to be contributed in hover climb as the high power lithium ion battery type is chosen for bat 1 . Bat 2 is used to fill up the mass budget and consequently overfulfills the power requirement.

Fig. 8 to Fig. 10 allow a more global perspective on the performance impact of the battery architectures. For different payload masses, the cruise endurance is given with respect to the cruise endurance of the SGL BAT architecture. The right figure depicts the used battery type combinations.

Fig. 8 uses the two aircraft with unchanged geometry parameters and excludes future battery technology. Generally, the TRI aircraft profits more from a dual battery architecture. Other than the QUAD, it cannot use high energy density cells with SGL BAT. Only the dual battery architecture enables to use the high energy cells. The battery architecture with the highest and most consistent benefits is BPMS BAT 2. The relative cruise endurance improvements are mostly within 10 to $20 \%$ for the TRI. Outliers peak to $40 \%$. TRI's dual use powertrain (respectively its usage of bat 1 in hover) promotes the more simple battery architectures SPRTD BAT and BAT MUX. The benefits of dual battery architectures tend to increase for lower payload mass respectively higher battery mass budget.

Fig. 9 as well uses the aircraft with unchanged geometry parameters, but includes the future battery in the battery choice. SGL BAT is not able to use the future battery type with superior energy density. Its power capability is too low even for the QUAD. For both aircraft types, dual battery architecture enable the access to the future battery type and achieve performance improvements of up to 35\%. Again, TRI can achieve higher benefits.

Fig. 10 optimizes the aircraft parameter for maximum cruise endurance and includes the future battery to the battery type selection. Due to the optimization, the usage of SGL BAT with battery type 2 is also possible for TRI. But still, battery type 1 is not useable with SGL BAT. Endurance improvements of 20 to $30 \%$ are well achieveable for both aircraft types when dual battery architectures are used together with battery type 1 .

Results will change with the addition of further battery types. SGL BAT will be affected more and to the better if an additional battery type can better meet the present combination of power and energy requirement. It also must be noted that not only high energy density, but also high power-density or intermediate battery types will improve in the future. With that, SGL BAT performance will enhance. But still, energy density will be compromised for those battery types. 

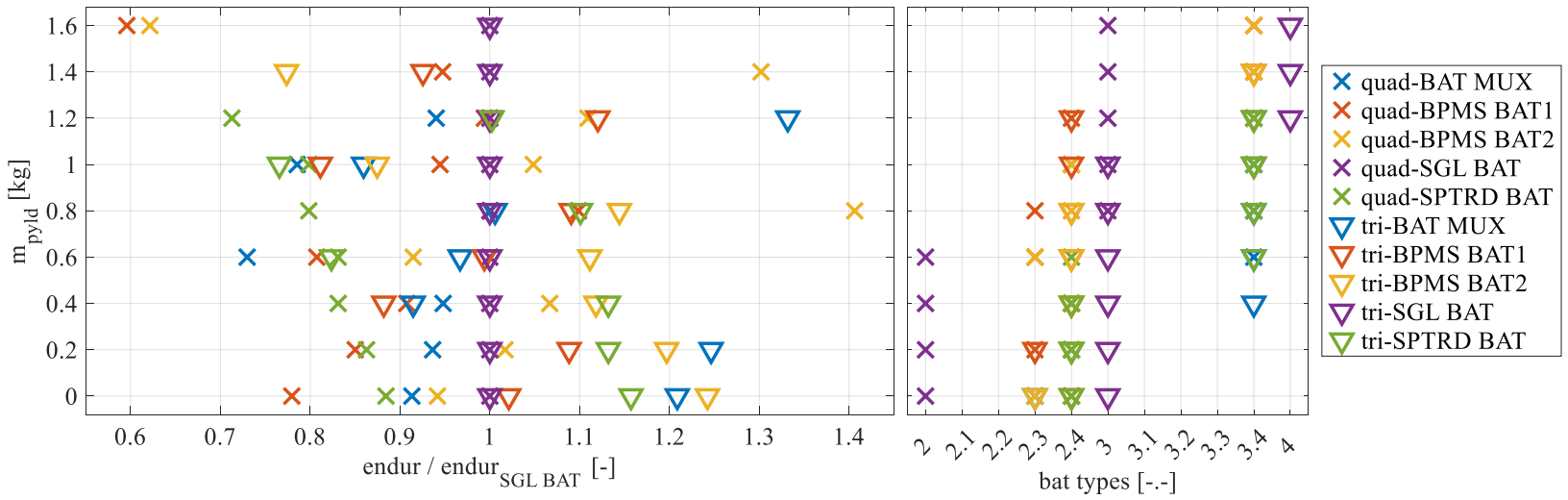

Fig. 8 Endurance gains for different battery architectures (same aircraft, without future bat type 1).

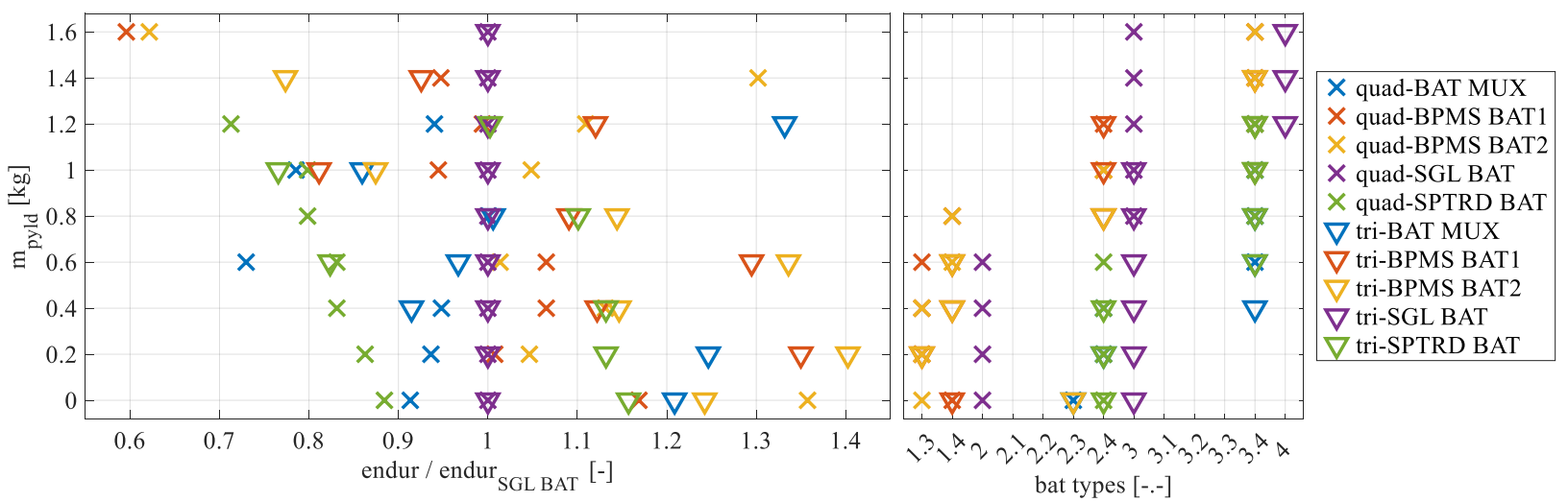

Fig. 9 Endurance gains for different battery architectures (same aircraft, with future bat type 1).
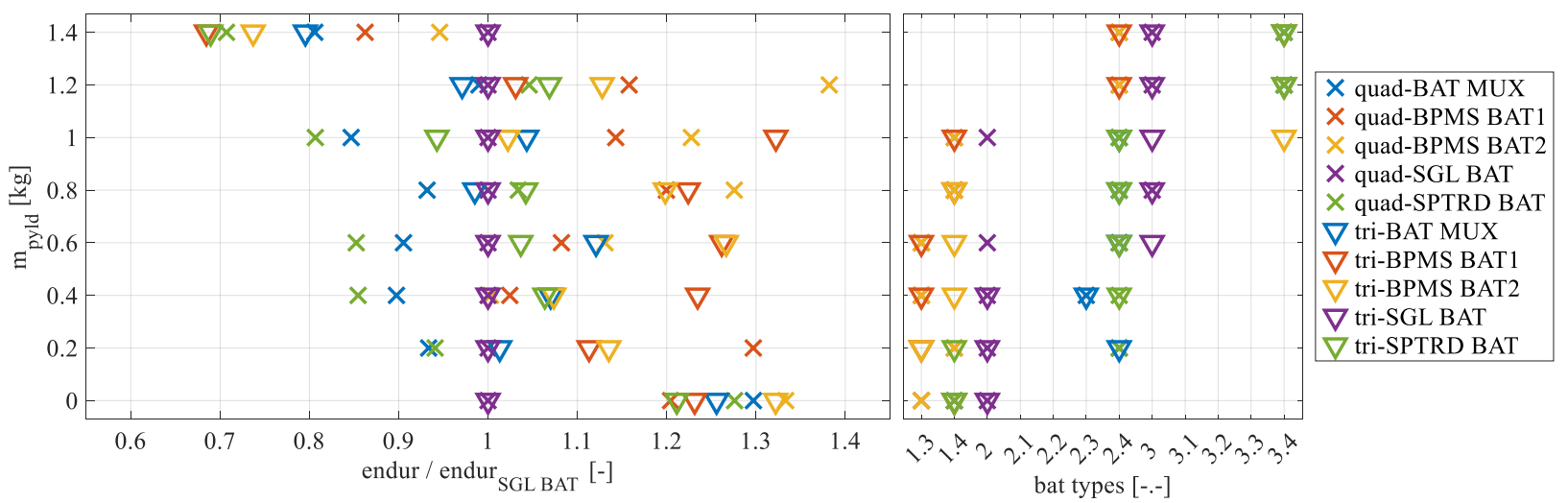

Fig. 10 Endurance gains for different battery architectures (optimized aircraft, with future bat type 1).

\section{Conclusion}

Dual battery architectures profit from two basic principles.

1) By using a dedicated battery for the short-time high power phase, the power requirement to the battery, which mainly supplies the long-duration low power phase, is relieved. A higher energy density battery can be used. Additional converters or switches however reduce the available mass for energy-carrying batteries. In summary, especially for high overall battery system mass budgets, the dual battery architecture provides more onboard energy leading to longer flight times in the low-power phase. This mechanism is more effective for fixed-wing VTOL aircraft configurations that sacrifice hover efficiency for cruise efficiency. If future 
battery technologies even more trade energy density against power capability, the usage of super energydense battery cells will only work with dual battery architectures.

2) The battery for the high power phase is available in significantly smaller mass increments than batteries with higher energy densities. The battery mass budget can be filled up better which increases onboard energy and flight time. This effect is promoted by a low ratio between overall battery mass budgets and single battery cell mass as well as high number of serial cell strings. Hence, small UAV are significantly affected. The battery architecture however must provide a crossfeed from the high power battery to the low power battery.

Within the conducted study for $5 \mathrm{~kg}$ aircraft, the benefit of a dual battery architecture significantly varies for the type of aircraft and payload mass. As a retrofit to an existing aircraft, dual battery architectures can improve the cruise endurance by 10 to $20 \%$ with current battery technology over a single battery architecture. This is consistently possible for an aircraft with high hover power consumption. Aircraft with very efficient hover merely show a benefit. Also the powertrain type is of relevance. Powertrains used in both hover and cruise promote the usage of especially simple dual battery architectures. Aircraft optimized from scratch together with the battery architecture reach 20 to $30 \%$ endurance increase over the single battery architecture. Here, the benefit arises from super high energy density battery types that get useable for fixed-wing VTOL aircraft by dual battery architectures.

The possible improvements in aircraft performance must be evaluated against operational drawbacks like reduced flexibility for missions with increased hover times or more effort in implementing and handling a second battery.

Dual battery architectures hold more potential to improve aircraft performance e.g. by inflight recharging or different voltage levels for the two batteries. To harvest the presented benefits in reality, the feasibility, especially of the most promising, but still generic battery architecture BPMS BAT 2 must be checked in detail.

\section{References}

[1] L. Gao, R. A. Dougal and S. Liu, "Power Enhancement of an Actively Controlled Battery/Ultracapacitor Hybrid," IEEE Transactions on Power Electronics, Volume 20, pp. 236-243, 2005.

[2] P. Stahl, C. Roessler and M. Hornung, "Performance and Life Cycle Cost Comparison of Optimized Fixed-Wing VTOL UAV Configurations," in Deutscher Luft- und Raumfahrtkongress 2019, Darmstadt, Germany, 2019.

[3] P. Stahl, T. Seren, C. Roessler and M. Hornung, "Development and Performance Comparison of Optimized Electric Fixed-Wing VTOL UAV Configurations," in ICAS Conference 2018, Belo Horizonte, Brazil, 2018.

[4] J. Gundlach, Designing Unmanned Aircraft Systems: A Comprehensive Approach, Blacksburg/VA: AIAA, 2012.

[5] Panasonic, "Specifications for Panasonic NCR18650GA," [Online]. Available: https://www.orbtronic.com/content/Datasheet-specs-Sanyo-Panasonic-NCR18650GA-3500mah.pdf.

[6] Sony Corporation, "Lithium Ion Rechargeable Battery Technical Information - US18650VTC5," August 2013. [Online]. Available: https://www.powerstream.com/p/us18650vtc5-vtc5.pdf.

[7] LaBatteria, "labatteria.com," 2111 2019. [Online]. Available: https://www.labatteria.co.il/en/e-bike-batterydischarge-rate-4c-vs-3c/.

[8] Oxis Energy Ltd., "Ultra Light Lithium Sulfur Pouch Cell Technology Specifications," April 2019. [Online]. Available: https://45uevg34gwlltnbsf2plyua1-wpengine.netdna-ssl.com/wp-content/uploads/2019/07/OXIS-LiS-Ultra-Light-Cell-spec-sheet-v4.2.pdf. 